\title{
Foco de doença de Chagas em São Fidélis, no Estado do Rio de Janeiro
}

\author{
Focus of Chagas disease in São Fidélis, in the State of Rio de Janeiro
}

\author{
Elias Seixas Lorosa ${ }^{1}$, Carolina Magalhães dos $\operatorname{Santos}^{1}$ e José Jurberg ${ }^{1}$
}

\begin{abstract}
RESUMO
Foram capturados quatro exemplares de Triatoma vitticeps e um de Panstrongylus geniculatus, no intradomicílio, no município de São Fidélis, Rio de Janeiro. Ficou comprovado a infecção positiva para Trypanosoma cruzi num dos exemplares de Triatoma vitticeps. Dois moradores da residência, um adulto e uma adolescente apresentaram sorologia positiva.
\end{abstract}

Palavras-chaves: Foco limitado. doença de Chagas. Triatominae.

\section{ABSTRACT}

Four specimens of Triatoma vitticeps and one of Panstrongylus geniculatus were caught inside a home in the municipality of São Fidélis, Rio de Janeiro. One of the specimens of Triatoma vitticeps proved positive for infection with Trypanosoma cruzi. Two people living in that home (one adult and one adolescent) presented positive serology.

Key-words: Limited focus. Chagas disease. Triatominae.

Em março de 2004, atendendo a solicitação de um morador do município de São Fidélis (latitude $21^{\circ} 38$ "46 sul, longitude $41^{\circ} 44$ " oeste, altitude $15 \mathrm{~m}$ em relação ao nível do mar), que fica localizado a $272 \mathrm{~km}$ da Cidade do Rio de Janeiro, uma equipe do Laboratório Nacional e Internacional de Referência em Taxonomia de Triatomíneos se deslocou para a região a fim de comprovar a existência de triatomíneos na casa. Esta fica localizada na área rural, próximo à mata, sendo sua construção de alvenaria, com reboco, coberta com telhas de barro, sem forro, tendo ao seu redor inúmeros anexos tais como galinheiro, chiqueiro, paiol e curral, além de cães e gatos.

Foram capturados e identificados cinco triatomíneos adultos, um macho e três fêmeas de Triatoma vitticeps (Stäl, 1859) e um macho de Panstrongylus geniculatus (Latreille, 1811), todos no intradomicílio.

No laboratório, através de técnica de precipitina, identificamos que três exemplares de Triatoma vitticeps tinham se alimentado em aves, roedores e ave/humano. Através do exame sorológico, dois moradores da casa, um homem com 41 anos de idade e uma adolescente com 14 anos, informaram terem sempre vivido na região, tiveram sorologia positiva para imunofluorescência indireta (IFI) e ensaio imunoenzimático (EIE) para doença de Chagas.
Estas duas espécies desde há muito são assinaladas para o Estado do Rio de Janeiro, Conorhinus vitticeps foi descrita por Stäl (1859) sem indicar se era Cidade ou Estado do Rio de Janeiro, em 1914 Neiva $^{9}$ confirma a presença da espécie infectada pelo Schizotrypanum cruzi no estado citando a localidade de Conceição de Macabu. Em 1922, Neiva e Pinto ${ }^{10}$ descrevem Triatoma fluminensis coletado em Japuiba, Angra dos Reis, mais tarde colocado em sinonímia por Pinto em 1931 como Triatoma geniculata, atualmente reconhecido como Panstongylus geniculatus $^{4}$. A literatura cita a distribuição geográfica de Triatoma vitticeps para três Estados a saber, Rio de Janeiro, Espírito Santos e Minas Gerais, enquanto Panstrongylus geniculatus tem vasta distribuição geográfica desde $20^{\circ}$ latitude Sul a $20^{\circ}$ latitude Norte, podendo ser encontrada até $1.600 \mathrm{~m}$ de altitude ${ }^{1}$.

Triatoma vitticeps e Panstrongylus geniculatus são espécies de hábitos tipicamente silvestres e eventualmente encontradas no intradomicílio; quando adultos são atraídos pela luz em casas próximas às matas ${ }^{56}$. Porém, no Estado do Espírito Santo já foi relatado o encontro de Triatoma vitticeps no intradomicílio, com suspeita de poder transmitir a doença de Chagas ${ }^{11}$. Já Panstrongylus geniculatus foi assinalado em ambiente peridomiciliar, no Estado do Pará, naturalmente

\footnotetext{
1. Laboratório Nacional e Internacional de Referência em Taxonomia de Triatomíneos, Instituto Oswaldo Cruz, Fundação Oswaldo Cruz, Rio de Janeiro, RJ. Apoio financeiro: Conselho Nacional de Desenvolvimento Científico e Tecnológico (CNPq), Chagas Disease Intervention Activities/Comunidade Européia (CDIA-CE), Convênio Secretaria de Vigilância em Saúde (SVS)/Fundação Oswaldo Cruz (FIOCRUZ - FIOTEC).

Endereço para correspondência: Dr. Elias Seixas Lorosa. Lab. Nac. Int. Ref. Taxonomia de Triatomíneos/FIOCRUZ. Av. Brasil 4365, Manguinhos, 21040-900 Rio de Janeiro, RJ. Telefax: $55212560-7317$

e-mail: lorosa@ioc.fiocruz.br

Recebido para publicação em 01/06/2007

Aceito em 15/7/2008
} 
infectado com Trypanosoma cruzi Z1, sugerindo um potencial para domiciliaçã $0^{13}$. Recentemente, Lorosa e cols $s^{6}$ encontraram um foco de doença de Chagas em Arcádia, Estado do Rio de Janeiro, cujo transmissor era Triatoma vitticeps.

A literatura assinala que Triatoma vitticeps pode colonizar as casas enquanto Panstrongylus geniculatus dificilmente o fará, pois seu ideal biológico chega próximo aos 100\% de umidade. Estas duas espécies devem ter sido atraídas pela luz e pelas condições adversas nos ecótopos naturais, constituindo-se um foco limitado e podem ser consideradas como vetores secundários em relação à transmissão da doença de Chagas ${ }^{128}$.

Até o presente, constatou-se que Triatoma vitticeps não coloniza o intradomicílio, mas sim o peridomicílio, o encontro de adultos nas casas deve-se à atração pela luz e pelas condições adversas do meio ambiente com a eliminação dos ecótopos naturais, esta espécie apresenta altas taxas de infecção pelo Trypanosoma cruzi $i^{32}$.

Após a constatação, a Secretaria de Vigilância em Saúde foi informada, voltando a casa, borrifou inseticidas mesmo não tendo encontrado mais triatomíneos. Anteriormente, os moradores da localidade já tinham notificado a FUNASA da existência de barbeiros na área.

\section{REFERÊNCIAS}

1. Carcavallo RU, Curto de Casas SI, Sherlock IA, Galíndez Girón I, JurbergJ, Galvão C, Mena Segura CA, Noireau F. Distribuição geográfica e dispersão altilatitudinal In: Carcavallo RU, Galíndez Girón I, Jurberg J, Lent $\mathrm{H}$ (eds) Atlas dos Vetores da Doença de Chagas nas Américas, Editora da Fundação Oswaldo Cruz, Rio de Janeiro, vol. 3, cap. 17, p.747-792, 1999.
2. Dias JCP. Controle de vetores da Doença de Chagas no Brasil e riscos de reinvasão domiciliar por vetores secundários. Memórias do Instituto Oswaldo Cruz 83 (supl 1): 387-391, 1998.

3. Diotaiuti L. Dinamica de poblaciones y control de Triatominos en Brasil. Actualización de la Tripanosomiasis Americana. In: Taller del Cono Sur, Universidad Nacional de Asunción, Paraguai p. 51-53, 2007.

4. Galvão C, Carcavallo RU, Rocha DS, Jurberg J. A checklist of the current valid species of the subfamily Triatominae Jeannel, 1919 (Hemíptera, Reduviidae) and their geographical distribution, with nomenclatyral and taxonomic notes. Zootaxa 202: 1-36, 2003.

5. Gonçalves TCM, Rocha DC, Cunha RA. Feeding patterns of Triatoma vitticeps in the state of Rio de Janeiro Brazil. Revista de Saúde Pública 34: 384-452, 2000.

6. Lorosa ES, Valente MMP, Cunha V, Lent H, Jurberg J. Foco de Doença de Chagas em Arcádia, estado do Rio de Janeiro-Brasil. Memórias do Instituto Oswaldo Cruz 98: 885-887, 2003.

7. Lent H. Transmissores da moléstia de Chagas no estado do Rio de Janeiro. Revista Fluminense de Medicina 7: 151-162, 1942.

8. Lent H, Wygodzinsky P. Revision on the Triatominae (Hemiptera, Reduviidae, Triatominae), and their significance as vector of Chagas' Disease. Bulletin of the American Musuem of Natural History 163: 127-520, 1979.

9. Neiva A. Presença em uma localidade do estado do Rio de Janeiro de um novo transmissor da moléstia de Chagas encontrado infectado em condições naturais (Nota previa). Brasilia Medica 28:333-335, 1914.

10. Neiva A, Pinto C. De um novo hemíptero brasileiro (Triatoma fluminensis nov. sp.). Brasilia Medica 36: 402-403, 1922.

11. Pinto AFS, Santos UM, Zaganelli FL, Almeida AZ, Carrancho PV. Doença de Chagas no estado do Espírito Santo VI - Observações sobre o Triatoma vitticeps, vetor do Trypanosoma tipo cruzi no município de Alfredo Chaves - ES. Revista Brasileira de Medicina Tropical 3: 53-54, 1969.

12. Souza RCM, Azeredo BUM, Lorosa E, Diotaiuti L. Infestação do ambiente artificial por Triatoma vitticeps no Município de Itanhomi, Minas Gerais, Brasil. In: Anais do Congresso da Sociedade Brasileira de Medicina Tropical, Belém, v. 38, p. 7, 2003.

13. Valente V. Potencial for domestication of Panstrongylus geniculatus (Latreille, 1811) (Hemiptera, Reduviidae, Triatominae) in the Municipality of Muaná, Marajó Island, State of Pará, Brazil. Memórias do Instituto Oswaldo Cruz 94 (supl I): 399-400, 1999. 